\title{
Simply-connected vortex-patch shallow-water quasi-equilibria
}

\author{
H. Plotka $\dagger$ and D. G. Dritschel \\ School of Mathematics and Statistics, University of St Andrews, St Andrews, Fife KY16 9SS, UK
}

(Received 9 May 2013; revised 31 October 2013; accepted 17 January 2014; first published online 5 March 2014)

We examine the form, properties, stability and evolution of simply-connected vortex-patch relative quasi-equilibria in the single-layer $f$-plane shallow-water model of geophysical fluid dynamics. We examine the effects of the size, shape and strength of vortices in this system, represented by three distinct parameters completely describing the families of the quasi-equilibria. Namely, these are the ratio $\gamma=L / L_{D}$ between the horizontal size of the vortices and the Rossby deformation length; the aspect ratio $\lambda$ between the minor to major axes of the vortex; and a potential vorticity (PV)-based Rossby number $R o=q^{\prime} / f$, the ratio of the PV anomaly $q^{\prime}$ within the vortex to the Coriolis frequency $f$. By defining an appropriate steadiness parameter, we find that the quasi-equilibria remain steady for long times, enabling us to determine the boundary of stability $\lambda_{c}=\lambda_{c}(\gamma, R o)$, for $0.25 \leqslant \gamma \leqslant 6$ and $|R o| \leqslant 1$. By calling two states which share $\gamma,|R o|$ and $\lambda$ 'equivalent', we find a clear asymmetry in the stability of cyclonic $(R o>0)$ and anticyclonic $(R o<0)$ equilibria, with cyclones being able to sustain greater deformations than anticyclones before experiencing an instability. We find that ageostrophic motions stabilise cyclones and destabilise anticyclones. Both types of vortices undergo the same main types of unstable evolution, albeit in different ranges of the parameter space, $(a)$ vacillations for large- $\gamma$, large-Ro states, (b) filamentation for small- $\gamma$ states and (c) vortex splitting, asymmetric for intermediate $-\gamma$ and symmetric for large- $\gamma$ states.

Key words: Rotating flows, Shallow water flows, Vortex dynamics

\section{Introduction}

The study of vortex equilibria, vortices which do not change in shape in an appropriately chosen frame of reference, is of fundamental interest in geophysical fluid dynamics. It provides insight into the nature of the long-lived vortical structures found not only in the oceans (Olson 1991; Carton 2001) and the Earth's and planetary atmospheres (Waugh \& Polvani 2010; Garate-Lopez et al. 2013), but also in simulations of geophysical turbulent flows (McWilliams 1984; Polvani, McWilliams, Spall \& Ford 1994). These coherent structures persist despite external factors, and often dominate late-time turbulent flows.

The equilibrium forms of vortices were first studied by Deem \& Zabusky $(1978 a, b)$, who considered one- and (translating) two-vortex configurations in the (barotropic)

$\dagger$ Present address: Department of Earth Sciences, ETH Zürich, 8092 Zürich, Switzerland.

Email address for correspondence: hanna@mcs.st-and.ac.uk 
two-dimensional Euler equations. Since then, a number of other studies have examined various equilibrium configurations in the barotropic context. Luzzatto-Fegiz \& Williamson $(2010,2011)$ considered a single-vortex (simply-connected) system and used an energy-based argument following Kelvin's variational principle (Thomson 1875) to find equilibrium solutions bifurcating from the Kirchhoff elliptical solution branch. Various two-vortex (doubly-connected) configurations have also been examined. Saffman \& Szeto (1980) and Pierrehumbert (1980) found solutions for symmetric corotating and translating states, respectively, while Dritschel (1995) examined asymmetric (in size) like- and opposite-signed vortices. Makarov \& Kizner (2011) further examined doubly-connected equilibria having an asymmetry in size and vorticities. Multiple-vortex equilibria have been studied by Dritschel (1985), who examined states composed of two to eight like-signed vortices.

A number of works have extended these barotropic studies, and examined how vortex equilibria are affected by a finite Rossby deformation length $L_{D}$, and the quasi-geostrophic shallow-water (QGSW) model is one of the simplest models in which it appears. Polvani (1988) and Polvani, Zabusky \& Flierl (1989) examined simply- and doubly-connected equilibria, and Płotka \& Dritschel (2012, 2013) also studied the stability of such states. Makarov, Sokolovskiy \& Kizner (2012) examined two-vortex like-signed doubly-symmetric equilibria, with each vortex in a different layer of a two-layer rotating fluid. Further studies, both experimental (Griffiths \& Hopfinger 1986, 1987) and numerical (e.g. Waugh 1992; Waugh \& Dritschel 1991; Yasuda 1995), have found that finite- $L_{D}$ effects result in qualitative changes in the behaviour of vortices.

The aforementioned studies all pertain to geostrophically-balanced systems, and therefore neglect aspects of realistic geophysical motions, which are not devoid of ageostrophic effects occurring at finite Rossby number. The study of vortex equilibria in an ageostrophic context is significantly more challenging. As a stepping stone for the highly complex, three-dimensional system, the two-dimensional shallow-water (SW) model may be considered. It is the simplest model which permits 'balanced' vortical motions, ageostrophic effects, and 'unbalanced' inertia-gravity waves (IGWs). Here, unlike in geostrophically balanced systems, the symmetry between anticyclonic and cyclonic motions, or motions in opposite directions (in the northern hemisphere, the clockwise and counterclockwise directions, respectively), is broken. This further allows insight into the well-known asymmetry which exists in both realistic geophysical flows (McWilliams 1985) and in their numerical simulations (Cushman-Roisin \& Tang 1990; Arai \& Yamagata 1994; Polvani et al. 1994, and others).

Despite being the simplest unbalanced model, the SW model still presents significant challenges for both analytical and numerical study. As a result, to date, only a few studies have examined SW equilibria. In fact, due to the spontaneous emission of IGWs, SW equilibria are not strictly steady, but radiate such weak IGWs that they may be deemed steady for all practical purposes. Cushman-Roisin, Heil \& Nof (1985) found exact analytical solutions for time-dependent cyclonic elliptical SW vortices, Cushman-Roisin (1987) did the same for anticyclonic ones, and Ripa (1987) developed a stability criterion for SW equilibria. However, this criterion is not applicable to isolated vortices. Stegner \& Dritschel (2000) numerically investigated the effects of size, the steepness of the vorticity profile and strength (Rossby number) on the stability of both cyclonic and anticyclonic isolated circular vortices. In the limit when the Rossby deformation length is large, Ford (1994) found peanut-shaped equilibria analogous to those found in the QGSW context by Polvani et al. (1989). 
Kizner, Berson \& Khvoles (2002) numerically constructed barotropic dipole equilibria, while Kizner et al. (2008) found exact, analytic solutions for steadily translating dipolar structures.

In this study, by using the SW model, we extend the work of Polvani et al. (1989) and Płotka \& Dritschel (2012), who studied dumbbell-shaped QGSW vortex-patch equilibria, which bifurcate from the Kirchhoff elliptical vortex (Kirchhoff 1876) at finite Rossby deformation lengths. In QGSW, the families of these states are completely spanned by two parameters: their aspect ratio $\lambda$, namely the ratio between their minor to major axes, and their horizontal size $L$ relative to the Rossby deformation length, $\gamma=L / L_{D}$. Note that $\gamma^{-2}$ is equivalent to the Burger number. In $\mathrm{SW}$, only one additional parameter, the Rossby number $R o$, is required. The Rossby number is a dimensionless measure of the strength of a vortex, defined by the ratio of the vortex potential vorticity (PV) anomaly $q^{\prime}$ to the Coriolis frequency $f, R o=q^{\prime} / f$. In balanced systems, $R o \equiv 0$; for cyclones $R o>0$, whereas anticyclones have $R o<0$. Our aim is to see to what extent the known QGSW equilibria are steady when extended into the SW context, and how their stability is affected by their size and strength. In addition, we aim to classify the types of instabilities near the boundary of stability, and to explore what role the ageostrophic component plays during instability. Note that in $\mathrm{SW} L_{D}=c / f$, where $c$ is the short-scale gravity wave speed and $f$ is the Coriolis frequency (twice the background rotation rate).

This paper is organised as follows. In $\S 2$ we describe the model formulation used and provide an overview of the numerical method and the flow initialisation procedure. In $\S 3$ we discuss how we quantify the degree of steadiness, while in $\S 4$ we present the extent to which the stability of the equilibria is affected by vortex strength and size, and in so doing address the cyclone-anticyclone asymmetry. Examples of the types of unstable evolution we find near the boundary of stability for various $\gamma$ and $R o$ are shown in $\S 5$. We end with some conclusions in $\S 6$.

\section{Model formulation}

Below, in $\S 2.1$, we start by describing the theoretical framework used in this study: the SW model. In $\S 2.2$ we discuss how we set up the flow in this system, providing details about the initial conditions used. Then, in $\S 2.3$ we give an overview of the contour advective semi-Lagrangian (CASL) numerical algorithm used for evolving the flow.

\subsection{The SW model and the concept of balance}

In this study, we use the full SW model (see e.g. Pedlosky 1979), which describes the motion of an incompressible fluid subject to planetary rotation and gravity effects. We use the single-layer, constant-density form of the model on the $f$-plane, which is expressed by the $\mathrm{SW}$ equations

$$
\begin{aligned}
\frac{\mathrm{D} u}{\mathrm{D} t}-f v & =-c^{2} \frac{\partial h}{\partial x}, \\
\frac{\mathrm{D} v}{\mathrm{D} t}+f u & =-c^{2} \frac{\partial h}{\partial y}, \\
\frac{\partial h}{\partial t}+\nabla \cdot((1+h) \boldsymbol{u}) & =0,
\end{aligned}
$$

where $\boldsymbol{u}=(u(x, y, t), v(x, y, t))$ is the (horizontal) velocity, $h(x, y, t)$ is the dimensionless fluid depth (or height) anomaly and $c=\sqrt{g H}$ is the short-scale 
gravity-wave speed, with $g$ being gravity and $H$ the mean fluid depth. The operator for the material derivative acting on a scalar $a$ is defined to be $\mathrm{D} a / \mathrm{D} t=\partial a / \partial t+\boldsymbol{u} \cdot \nabla a$.

The flow described by the SW equations may be decomposed into two parts: the dominant, low-frequency 'balanced' component relating to vortical motions, and a faster, relatively short-lived 'unbalanced' component consisting of ageostrophic motions such as IGWs. It is beneficial to view the balanced, vortical motions as local concentrations of $\mathrm{PV}$, a scalar, which in the absence of viscous and diabatic effects is materially conserved by fluid particles. In SW, the PV $q$ is defined to be

$$
q=\frac{\zeta+f}{1+h},
$$

where $\zeta=\partial v / \partial x-\partial u / \partial y$. The statement of conservation of $\mathrm{PV}, \mathrm{D} q / \mathrm{D} t=0$, is a direct result of (2.1), and can also be made for its anomaly relative to a constant background value, $q^{\prime}=q-f$. In QGSW, in which the unbalanced component has been completely filtered out, it is possible to determine exactly all dynamical fields from the instantaneous distribution of (quasi-geostrophic) PV. In SW, the exact separation of a flow into a balanced and an unbalanced component is not possible, as the "slow manifold' completely devoid of IGWs does not exist (Ford, McIntyre \& Norton 2000). Instead it makes sense to speak of 'minimally-unbalanced' flows, which are set up in a way to contain as few IGWs as possible. For such minimally-unbalanced flows it is no longer possible to determine exactly the dynamical fields from the distribution of PV. Nevertheless, through what is known as the 'invertibility principle' (Hoskins, McIntyre $\&$ Robertson 1985) various, sometimes very accurate, methods for approximating them do exist (McIntyre \& Norton 2000; Mohebalhojeh \& Dritschel 2000; Mohebalhojeh 2002; Dritschel \& Viúdez 2003; McKiver \& Dritschel 2008, and others). These enable one to estimate the ageostrophic part of the flow arising from PV, as well as the proportion of IGWs in a flow.

\subsection{Flow initialisation}

The solutions of the SW equations can describe a variety of realistic geophysical motions. Here, we seek those which represent relative vortex equilibria (referred to as just 'equilibria'). We do this by making use of the simply-connected two-fold symmetric relative equilibria generated by Polvani et al. (1989) and Płotka \& Dritschel (2012). In the QGSW model of the aforementioned studies, these states consist of a patch of constant QGPV in a background of zero PV, and are fully described by two parameters: their size relative to the Rossby deformation length, $\gamma$, and their aspect ratio, $\lambda$. In SW, only one additional parameter, the Rossby number $R o$, is necessary to describe the vortex-patch equilibria. The PV anomaly inside the vortex patch is then defined in terms of the Rossby number by $q^{\prime}=R o f$.

We generate SW equilibria from QGSW ones (formally at $R o=0$ ) by using a modification of the 'optimal PV balance' procedure of Viúdez \& Dritschel (2004), in which the PV anomaly is ramped up to a prescribed value over a sufficiently long ramping period (here taken to be $10 T_{i p}$, where $T_{i p}=2 \pi / f$ is the inertial time period). Further details of the flow initialisation are given in the Appendix.

Note that as mentioned in the introduction, there is always a small amount of IGW radiation from SW vortices (see Ford et al. 2000, and references therein for a brief review of the topic of spontaneous gravity wave emission), so the states we generate are not strictly equilibria, but rather quasi-equilibria. However, as discussed in the next sections, by initialising the flow with only minimal amounts of imbalance, it is still 
useful to view the states as equilibria. Here, we call our states 'equilibria' in order to avoid the more cumbersome 'quasi-equilibria' term.

In order to provide an overview of the parameter space of realistic flows, we generate equilibria for both small and large $\gamma$, namely for $\gamma=0.25,1,2,3,4$ and 6 (this gives a range of Burger numbers between 16 and 0.028). We examine both cyclonic $(R o>0)$ and anticyclonic $(R o<0)$ states with Rossby numbers $|R o| \leqslant 1.0$ at $\Delta R o=0.1$.

\subsection{Flow evolution}

Having generated equilibria with non-zero Ro, we solve the SW equations using the CASL algorithm (Dritschel \& Ambaum 1997; Dritschel, Polvani \& Mohebalhojeh 1999). The algorithm keeps track of PV contours (across which $q$ jumps by a prescribed increment $\Delta q$ ), and of the velocity and height field on a grid. Note that here we have only one contour defining the boundary of the equilibrium, with the jump $\Delta q=q^{\prime}=R o f$. To improve the accuracy of simulating both the balanced and unbalanced components of the flow, a variable transformation is made from the standard set $(u, v, h)$ to $(q, \delta, \chi)$, where $\delta$ and $\chi$ are the divergence of the velocity and acceleration, respectively. Note that on the $f$-plane, $\chi / f$ is the ageostrophic vorticity. The primitive variables are then recovered from $(q, \delta, \chi)$ via an inversion procedure so that PV contours can be advected, and the field tendencies calculated. The variable transformation results in a demonstrably more accurate representation of IGWs and vortical motions (Mohebalhojeh \& Dritschel 2000; Smith \& Dritschel 2006). We evolve $q$ using contour advection, while $\delta$ and $\chi$ are evolved using standard pseudo-spectral methods in a square doubly-periodic domain of side length $2 \pi$ at a resolution of $256^{2}$. The time stepping is carried out using a fourth-order Runge-Kutta scheme, with a time step chosen to marginally resolve the fastest gravity wave, i.e. $\Delta t=\Delta x / c$, where $\Delta x$ is the grid spacing.

We use the CASL algorithm to evolve members of the families of equilibria for a range of $\gamma$ and $R o$. We aim to determine the critical aspect ratio $\lambda_{c}=\lambda_{c}(\gamma, R o)$ at which the equilibria cease to be stable, and to describe their unstable evolution near the boundary of stability. As a first guess for $\lambda_{c}$ at $|R o|=0.1$, we use the known QGSW value of $\lambda_{c}(\gamma, 0)$ determined by Płotka \& Dritschel (2012). Increasing the Rossby number by a small amount only slightly modifies the form of the equilibria, and so too (it is found) the location of the boundary of stability. Once we determine $\lambda_{c}(\gamma,|R o|=0.1)$, we continue to seek equilibria for progressively larger $R o= \pm 0.1$, using $\lambda=\lambda_{c}(\gamma, R o-0.1)$ (for cyclones) or $\lambda=\lambda_{c}(\gamma, R o+0.1)$ (for anticyclones) as a guess for $\lambda_{c}(\gamma, R o)$. We determine the location of $\lambda_{c}$ within an accuracy of $\Delta \lambda=$ 0.005 .

We evolve each steady state for at least 100 particle rotation periods $T_{p}$ after the ramping of the $\mathrm{PV}$ anomaly has been completed. One $T_{p}$ is the amount of time taken by a fluid particle to circuit the boundary of the equilibrium. In QGSW, $T_{p}^{Q G S W}=2 \pi / \Omega_{p}$, where $\Omega_{p}$ is the particle rotation rate of the equilibrium, the values of which are shown in figure 1 in the $\gamma-\lambda$ parameter plane, as calculated by Płotka \& Dritschel (2012). In SW, increasing the Rossby number increases particle velocities, and so we scale $T_{p}$ accordingly by setting $T_{p}=T_{p}^{Q G S W} / R o$.

\section{Quantifying the degree of steadiness}

The long-time persistence of the SW equilibria we generate testifies to their steadiness, and thus their stability. However, we seek a more quantitative measure to 


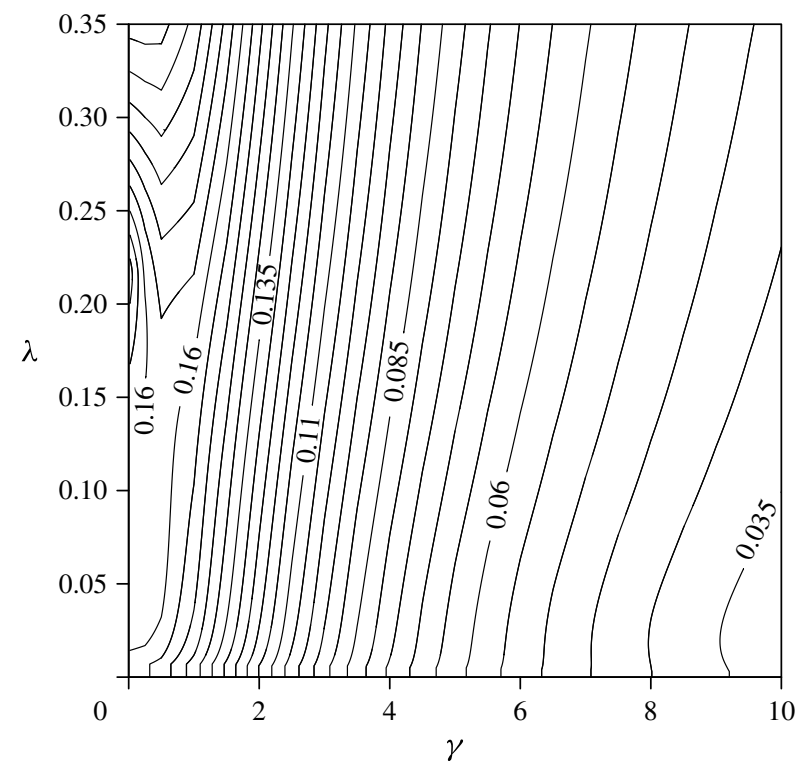

FIGURE 1. The particle rotation rate $\Omega_{p}$ (contour interval 0.005) of QGSW equilibria in the $\gamma-\lambda$ parameter plane. This figure is reproduced from Płotka \& Dritschel (2012, figure $6(d))$.

verify this, and begin by noting that the Bernoulli pressure $B$ and the PV are both functions of the streamfunction for steady (or steadily rotating) solutions of the SW equations (Malanotte-Rizzoli 1982; Baey \& Carton 2002; Kizner et al. 2008). This means that in a steady flow, $B$ is a constant on contours of PV, for an appropriately chosen frame of reference. In rotating SW, the Bernoulli pressure function takes the form

$$
B=h c^{2}+\frac{1}{2}\left|\boldsymbol{u}^{\prime}\right|^{2}-\frac{1}{2} \Omega f\left(x^{2}+y^{2}\right),
$$

with the velocity in the rotating reference frame $\boldsymbol{u}^{\prime}=(u+\Omega y, v-\Omega x)$ and $\Omega$ the rotation rate. Note that $B$ reduces to the quasi-geostrophic streamfunction $\psi$ multiplied by $f$ in the limit $R o \rightarrow 0$. Note that the rotation rate can be calculated following Dritschel (1995) by minimising the variance of the normal velocity along the PV contour. Figure 2 shows an example of the extent to which the contours of constant $B$ (shown in grey) and PV (shown in black) are aligned for a steady, stable state $(a)$ and an unstable one $(b)$ near $\lambda=\lambda_{c}$. We see that for the stable state, the contours closely correspond, while crossing is apparent in the unstable one.

We check how close we are to a steady solution of (2.1) by defining the nondimensional steadiness parameter $\epsilon_{b}$, which measures how closely aligned the contours of $\mathrm{PV}$ and $B$ are in a reference frame rotating with the equilibrium:

$$
\epsilon_{b}=\frac{\sqrt{\frac{1}{P} \oint_{\mathscr{C}}(B-\bar{B})^{2} \mathrm{~d} s}}{\bar{B}},
$$

where $P=\oint_{\mathscr{C}} \mathrm{d} s$ is the perimeter of the PV contour $\mathscr{C}$, and $\bar{B}=(1 / P) \oint_{\mathscr{C}} B \mathrm{~d} s$ is the mean Bernoulli pressure around the PV contour. Figure 3 shows how $\epsilon_{b}$ changes through time for both stable, anticyclonic states $(a)$ and unstable ones $(b)$ near the 
(a)

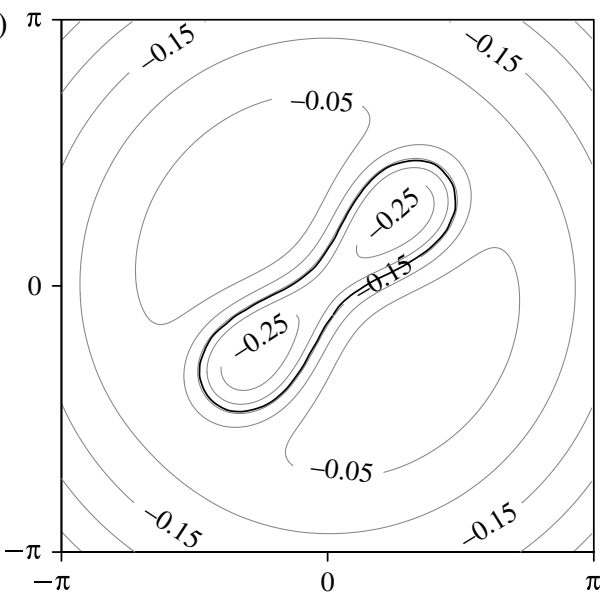

(b)

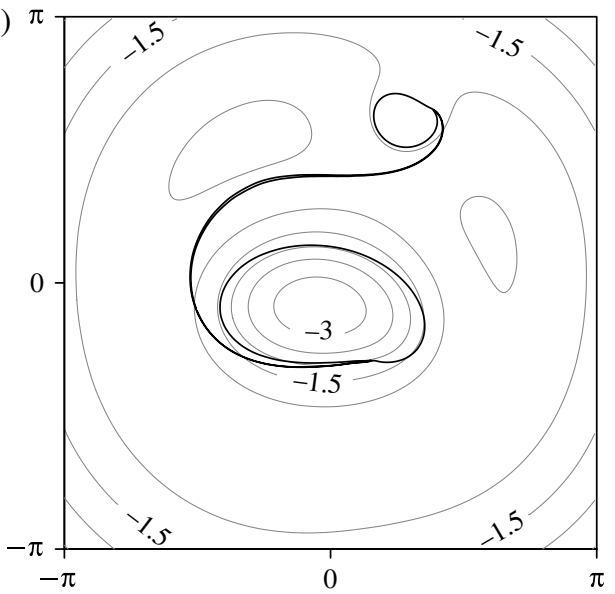

FIGURE 2. PV contours (black) and contours of the Bernoulli pressure function (grey) in the $x-y$ plane. We see $(a)$ a stable state with $(\gamma, R o)=(3,0.1)$ at $t=53.08 T_{p}$ (contour interval 0.1$)$ and $(b)$ an unstable state with $(\gamma, R o)=(1,0.3)$ at $t=25.97 T_{p}$ (contour interval 0.5$)$, both near $\lambda=\lambda_{c}$.
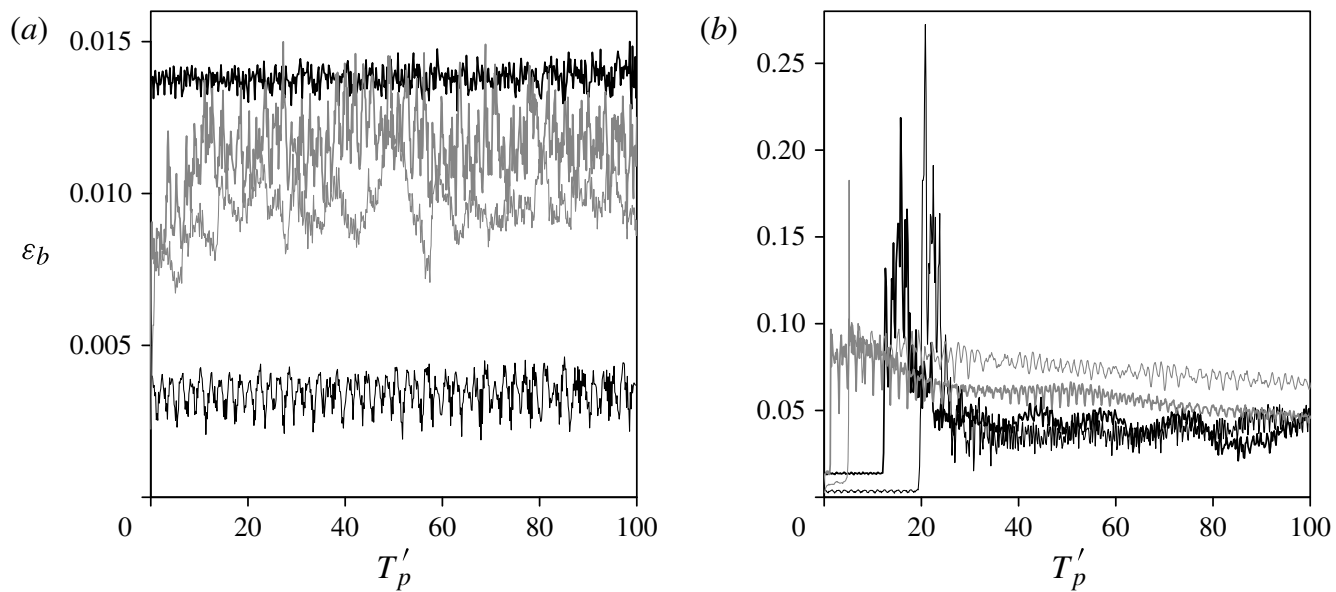

FIGURE 3. The value of $\epsilon_{b}$ as a function of time. Here, we show $\gamma=0.25$ (black) and $\gamma=6$ (grey) for $R o=-0.1$ (thin lines) and $R o=-0.5$ (bold lines) near the boundary of stability for steady, stable states $(a)$ and unstable ones $(b)$. We show how the flow develops for $100 T_{p}$ once the PV ramping period has been completed, so ' $t=0$ ' occurs at the end of the ramping period.

boundary of stability. In each panel of the figure, we show how $\gamma$ and Ro affect the value of $\epsilon_{b}$ by examining states having $\gamma=0.25$ at $R o=-0.1$ and -0.5 with black thin and bold lines, respectively, and for states having $\gamma=6$ at the same $R o$ with grey thin and bold lines. We see that the $\epsilon_{b}$ values are consistently small for both the cyclonic and anticyclonic stable states, but also initially in the unstable states. In addition, for the stable states, changing the Rossby number does not affect the values of the steadiness parameter at $\gamma=6$, whereas even though $\epsilon_{b}$ remains small for all time, the effects of increasing $|R o|$ are noticeable at $\gamma=0.25$ (we return to this 
point in §4). On the other hand, the effects of $|R o|$ are not visible in the unstable states, where the value of $\epsilon_{b}$ instead seems to depend on $\gamma$, with small- $\gamma$ vortices having larger $\epsilon_{b}$ values than large- $\gamma$ vortices. This mainly results from the difference in the types of unstable evolution small and large vortices undergo (see §5). After an instability has taken place, the flow returns to a quasi-steady state, with the value of $\epsilon_{b}$ falling again. This return to steadiness is quicker and more pronounced at small $\gamma$, but a downward trend is also visible for states at higher $\gamma$.

Throughout this paper we refer to states which are 'steady', and ones which are 'stable' and 'unstable'. After the initial PV ramping procedure is completed, the values of $\epsilon_{b}$ for all of the states considered in this study are low. This is true for both the 'stable' and 'unstable' states (cf. figure 3, especially at early times for the unstable states). For the 'stable' ones, $\epsilon_{b}$ stays small for the entire duration of the simulation, while for the 'unstable' states there is a sudden spike in $\epsilon_{b}$ at some time $t$, during which the vortex undergoes unstable evolution. We do not attempt to perform a formal linear stability analysis of our equilibria, as doing so in SW is extremely difficult. However, we do determine a boundary of 'stability' at $\lambda=\lambda_{c}$, such that for $\lambda>\lambda_{c}$, if left undisturbed, the vortex persists for long times without change in shape, and for $\lambda<\lambda_{c}$ it undergoes unstable evolution. Hence, when 'stable' states are referred to in this paper, these are states which do not change in shape throughout the (sufficiently long) duration of the simulation, while 'unstable' states are ones which do change in shape significantly.

The steadiness parameter $\epsilon_{b}$ is a good measure for determining the critical aspect ratio $\lambda_{c}$ at which there is a sharp spike in $\epsilon_{b}$. However, for states with $\gamma>1$, through a visual examination of the equilibria, we find weakly-unstable states for which $\epsilon_{b}$ has low values for all time. Unlike the states having $\lambda<\lambda_{c}$, these states do not undergo major changes in shape, and instead exhibit quasi-steady vacillations, during which their aspect ratio goes through cycles of increasing and decreasing its value. This process is sufficiently slow that the Bernoulli pressure $B$ is able to adjust itself about the vortex, and no signal of the weak instability is apparent in $\epsilon_{b}$. We introduce the vacillating aspect ratio $\lambda_{v}$, such that for any $\lambda_{c}<\lambda<\lambda_{v}$ the state undergoes vacillations, while for $\lambda \geqslant \lambda_{v}$ it is stable. Determining the exact location of $\lambda_{v}$ is difficult, as for $\lambda$ ranging from $\lambda_{c}$ to $\lambda_{v}$ the change in aspect ratio during one vacillation cycle, $\Delta \lambda_{c y c}$, decreases. We define $\lambda_{v}$ to be the smallest aspect ratio at which, for an appropriate choice of $b, \Delta \bar{\lambda}_{c y c} / \lambda_{i}<b$, where $\lambda_{i}$ is the initial aspect ratio of the state. We take the average of $\Delta \lambda_{c y c}$ throughout the simulation, $\Delta \bar{\lambda}_{c y c}$, and scale it by $\lambda_{i}$ to account for the wide range of $\lambda_{i}$ considered. We set $b=0.05$ since in SW even balanced, steady states generate a small amount of IGWs, and hence there is always some deformation to the shape (and mass) of the state. We have deemed that an average change of less than $5 \%$ in the initial aspect ratio is sufficiently small for a state to be termed steady. In addition, as $\lambda_{v}$ only exists for states with $\gamma>1$, we introduce the steady aspect ratio $\lambda_{s}$ such that all states with $\lambda>\lambda_{s}$ are steady; then $\lambda_{s}=\lambda_{c}$ when vacillations do not occur, and $\lambda_{s}=\lambda_{v}$ when they do. The location of $\lambda_{c}$ and $\lambda_{v}$ in the parameter space considered is discussed in detail in $\S 4$, of which a summary is presented in figure 6. However, before discussing the stability of the equilibria, we first examine a few of their properties.

At a fixed $(\gamma, R o)$, figure 4 shows $\bar{\epsilon}_{b}$, the time average of $\epsilon_{b}$ (over the duration of a simulation) for the stable states near $\lambda_{s}$. We see that in general, at a fixed $\gamma$, states with smaller $|R o|$ have lower values of $\bar{\epsilon}_{b}$ than do states with larger $|R o|$. This is to be expected, as the amplitude of the free-surface deformations caused by the vortex increases with $|R o|$. The cyclone-anticyclone asymmetry is also apparent 


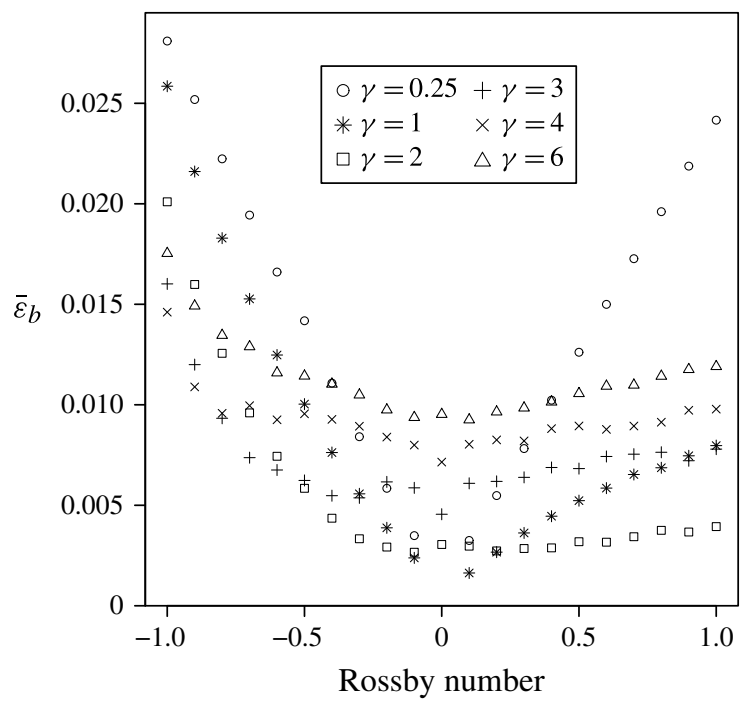

FIGURE 4. The time average (over the duration of the simulation) of the steadiness parameter, $\bar{\epsilon}_{b}$, at a fixed $(\gamma, R o)$ for the range of $\gamma$ and $R o$ considered. Here, we show the average of $\bar{\epsilon}_{b}$ for stable states with $\lambda-\lambda_{s}<0.020$. The values at $R o=0$ are obtained from evolving QGSW states with a quasi-geostrophic version of CASL.

from the figure, where for all $\gamma$, at a fixed $|R o|$, cyclones have lower values of $\bar{\epsilon}_{b}$ than anticyclones. This asymmetry is strongest for small- $\gamma$ states, and weakens as $\gamma$ increases for small $|R o|$. In addition, we see that for large- $\gamma$ cyclonic states, the value of $\bar{\epsilon}_{b}$ becomes $R o$-independent, while for anticyclones there is a strong variation with Ro. Finally, we note that when the QGSW states are evolved using a doubly-period quasi-geostrophic version of CASL, despite being balanced, they too have non-zero values of $\overline{\epsilon_{b}}$ which grow with $\gamma$ (shown for $\gamma>1$ ). This residual unsteadiness is the result of placing the vortex in a finite periodic box and using a much lower effective resolution than afforded by the contour dynamics method used in the QGSW study (Płotka \& Dritschel 2012).

In figure 5 we show $M / H$, where

$$
M=H \iint_{\mathscr{D}}(1+h) \mathrm{d} x \mathrm{~d} y,
$$

is the mass contained within the equilibria at the end of the PV ramping period, as a function of $R o$ for the range of $\gamma$ considered near $\lambda=\lambda_{s}$. For simplicity, we only describe the anticyclonic case, though the same arguments can be made for cyclones which have a negative, rather than positive, depth anomaly $h$, and at small $|R o|$ are antisymmetric to the anticyclonic states. We see that for the anticyclonic states, as $|R o|$ increases, so does the amount of mass contained within the vortex, as $h$ also increases. In addition, at a fixed $R o$, vortices with larger $\gamma$ contain greater mass. Larger vortices (having larger $\gamma$ ) also feel the effects of increasing $|R o|$ more. The mass contained by the $\gamma=0.25$ equilibria at $R o=-0.1$ and $R o=-0.6$ differs by around $1 \%$, whereas for the $\gamma=6$ equilibria this difference is about $36 \%$.

We note that for both cyclones and anticyclones, at small $|R o|$, there appears to be a linear relationship between $M$ and $R o$. We attempt to explain this as follows. 


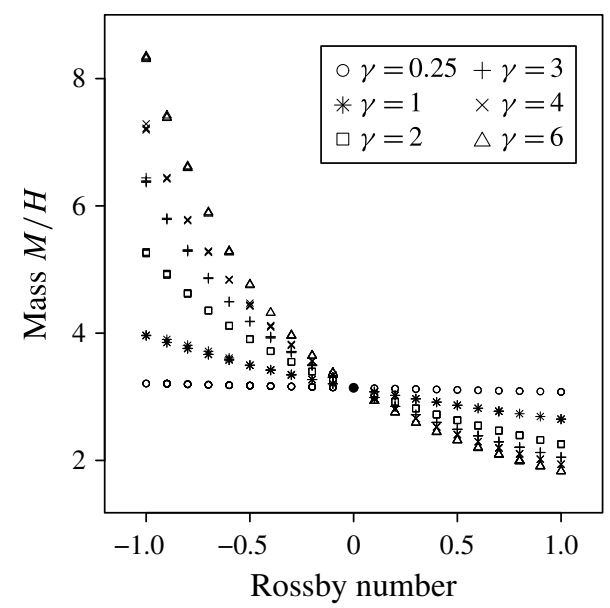

FIgURE 5. The mass $M / H$ of the equilibrium at the end of the PV ramping period, shown as a function of $R o$ for the steady states near $\lambda=\lambda_{s}$. The filled in point indicates the QGSW mass $M=\pi H$.

It is known that in SW, near the quasi-geostrophic limit (for small $|R o|$ ), the mass anomaly (the difference from the QGSW mass) scales like $m^{\prime} \sim \mathrm{Fr}^{2} /$ Ro (see e.g. Vallis 2008), where $F r=U / c$ is the Froude number, with $U$ being the characteristic horizontal velocity scale. For large $\gamma$, the maximum speed on the vortex edge scales like $U \sim R o f L_{D}$ (Nycander, Dritschel \& Sutyrin 1993). Hence, since $c=f L_{D}, F r \sim R o$ which implies $m^{\prime} \sim R o$ as observed. We find in fact that this scaling holds for all of the $\gamma$ considered (including $\gamma<1$ ), and for values of $|R o|$ up to 0.4 . The departures from this linear relationship are less than 0.07 at large $\gamma$ and up to two orders of magnitude smaller at small $\gamma$. Note that the amount of mass added (subtracted) by anticyclones (cyclones) is not symmetric, and at a fixed $|R o|$, anticyclones contain more mass than cyclones are able to displace. Finally, we note that as we are considering quasi-equilibria which are not strictly steady, small fluctuations in mass do occur for the stable states during evolution. However, these are $\ll 1 \%$ of the total mass for states with $\gamma \lesssim 3$ and no more than $2.4 \%$ for large- $\gamma$ states.

\section{Stability}

We next discuss how the stability of the equilibria discussed above varies with $\gamma$ and $R o$, and thus study the naturally-occurring asymmetry between cyclonic and anticyclonic vortices. We say that a vortex is stable (sometimes also weakly unstable) when it undergoes no major structural changes for at least $100 T_{p}$ after the end of the PV ramping period. We measure this via the steadiness parameter $\epsilon_{b}$, in which a clear jump is visible during the onset of instability (cf. figure 3) providing us with the location of the critical aspect ratio $\lambda_{c}(\gamma, R o)$. In addition, at larger $\gamma$, we find the location of the vacillating aspect ratio $\lambda_{v}(\gamma, R o)$, and thus find $\lambda_{s}(\gamma, R o)$, such that at a fixed $(\gamma, R o)$, all states having $\lambda \geqslant \lambda_{s}$ are stable. The different types of unstable behaviour are discussed in the next section, and here we focus on the location of the boundary of stability $\lambda_{s}(\gamma, R o)$. Note that at some $(\gamma, R o)$ only one of $\lambda_{c}$ or $\lambda_{v}$ may exist.

Figure 6 shows the location of $\lambda_{c}$ (marked by filled-in circles in the figure) and $\lambda_{v}$ (marked by empty circles) as a function of $\operatorname{Ro}$ for various $\gamma$. We also indicate states 

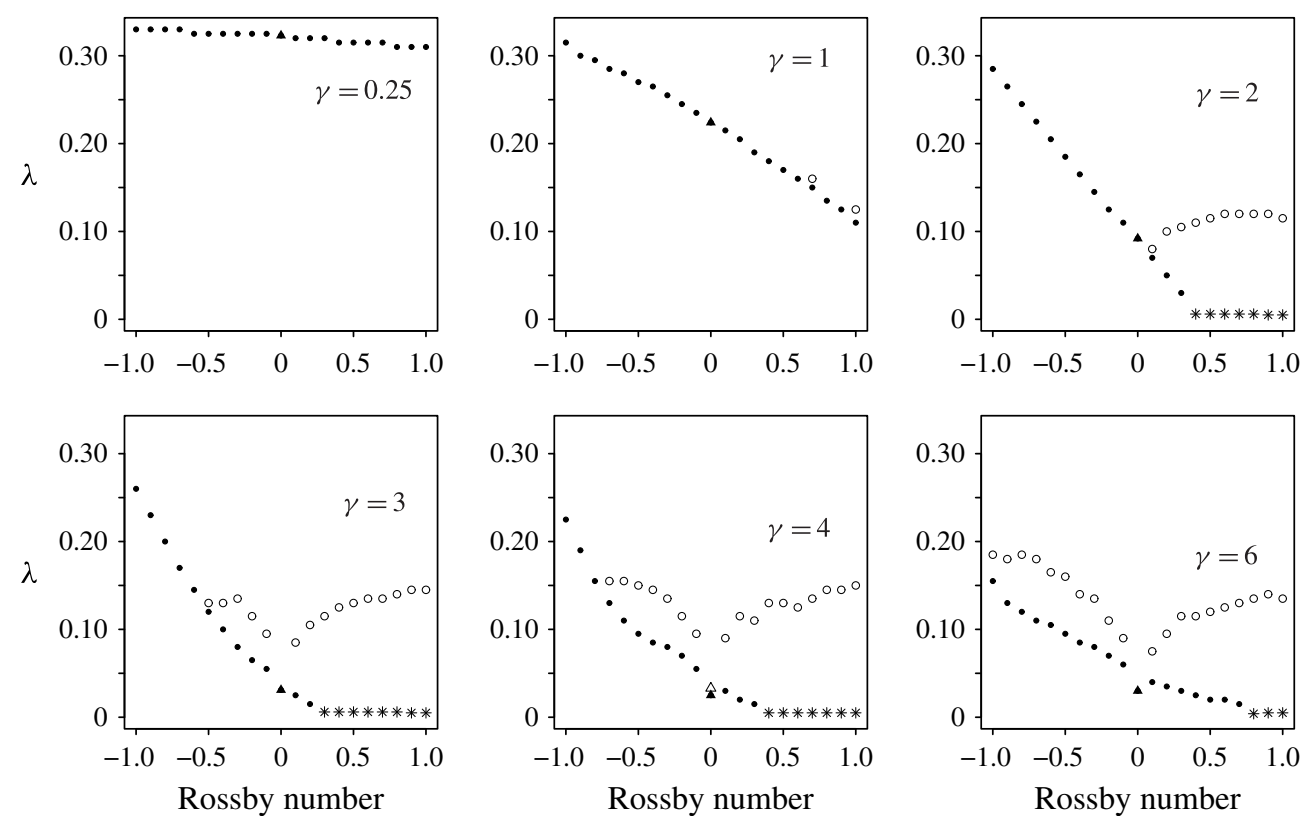

FIgURE 6. The location of $\lambda_{c}$ (filled-in circles) and $\lambda_{v}$ (empty circles) as a function of $R o$ for various $\gamma$, as labelled. The filled-in triangle in each plot shows the location of the QGSW $\lambda_{c}$, and the asterisk indicates a vacillating state with the smallest $\lambda$ we have been able to generate, which is weakly-unstable. The triangle in the $\gamma=4$ panel marks the location of $\lambda_{c}$ found by the quasi-geostrophic CASL code. We determine $\lambda_{c}$ and $\lambda_{v}$ to within $\Delta \lambda=0.005$.

for which we do not find $\lambda_{c}$ (marked by asterisks). These results offer the clearest picture of the cyclone-anticyclone asymmetry. Cyclones are generally more stable in that they remain stable for smaller $\lambda$ than anticyclones. The difference is immediate even at small $R o$.

For $\gamma \lesssim 2$, the location of $\lambda_{c}$ varies nearly linearly with $R o$, and includes the QGSW limit $\lambda_{c}(\gamma, 0)$ found by Płotka \& Dritschel (2012). For larger $\gamma$ however, the QGSW limit value appears to depart from the collection of nearby points with $R o \neq 0$. That is, the QGSW state at $R o=0$ can become noticeably more deformed before losing stability than nearby small $|R o|$ states. The effects of a doubly-periodic domain and finite resolution provide perturbations leading to instability which are absent in the QGSW study, in which an infinite domain and much higher effective resolution was used. As discussed in $\S 3$, when the QGSW states are placed in a periodic box at finite resolution and evolved using a quasi-geostrophic version of CASL, the average steadiness parameter $\bar{\epsilon}_{b}$ is non-zero and increases with $\gamma$. In fact, for large $\gamma$, using a finite resolution to evolve the QGSW states leads to a small increase in $\lambda_{c}$, bringing it into line with the values found at small Ro for the SW states, as shown by the triangle for $\gamma=4$ in figure 6 .

From figure 6 we also see that at large $\gamma$ there is a change in slope in the line fitted through the values of $\lambda_{c}$ for anticyclonic states, where it is shallower for small $|R o|$, and steeper for large $|R o|$. For example, at $\gamma=4$, the slope of the line predicting the location of $\lambda_{c}$ for $|R o| \lesssim 0.5$ is -0.11 , while for $|R o| \gtrsim 0.5$ it is -0.29 . For larger $\gamma$, this change occurs at higher $|R o|:$ at $\gamma=3$, it happens at $R o \approx-0.5$, while for 
$\gamma=6$, at $R o \approx-0.9$. If we once again compare this behaviour to the results presented in figure 4 , we see that at large $\gamma$, for states with $R o \lesssim-0.5$ the slope of the line fitted through the $\bar{\epsilon}_{b}$ values steepens compared with the slope of the line fitted through values with $R o \gtrsim-0.5$. Higher values of $\bar{\epsilon}_{b}$ mean that states become less steady, which in turn explains why the slope of the line through $\lambda_{c}$ steepens. We do not see this behaviour in cyclonic states, where the slope of the line fitted through $\bar{\epsilon}_{b}$ values does not change. However, for large- $\gamma$ cyclones, at large $R o$, strong instabilities cease to occur. The value of $R o$ at which this happens increases with $\gamma$.

For small- $\gamma$ states, the location of $\lambda_{c}$ coincides with the location $\lambda_{s}$, in contrast to large- $\gamma$ states $(\gamma \gtrsim 1)$, where a region of weakly unstable states occurs. For $\gamma=2$, vacillations only occur for cyclonic states, and although variations in the exact location of $\lambda_{v}$ at different $R o$ are small, it appears that states with $R o \lesssim 0.6$ need to increase their aspect ratios for stability, and states with $R o \gtrsim 0.6$ can once again decrease it while retaining their stability. As $\gamma$ increases, vacillations occur not only for smaller values of $R o$, but also for a wider range of $\lambda$. In fact, for $\gamma \geqslant 2$, at large $R o>0.4$ this is the only type of instability which occurs. For cyclones, across all $\gamma$, the vacillating region expands as Ro increases, and states need to be less deformed in order to be stable. The same is true for anticyclones at small $|R o|$, but as $|R o|$ increases, for large enough $\lambda_{c}$ no vacillations occur. This is most visible for $\gamma=3$, where for $-0.5 \leqslant R o \leqslant-0.3$ the vacillating aspect ratio $\lambda_{v} \approx 0.130$, and as $|R o|$ increases it converges to $\lambda_{c}$. Similarly as with $\lambda_{c}$, at large $\gamma$, the location of $\lambda_{v}$ appears to be converging towards $\lambda_{c}(\gamma, 0)$ in a nonlinear way. Note that apart from at certain $\lambda$ values at $\gamma=5$ and 6, Płotka $\&$ Dritschel (2012) found no evidence for vacillations in QGSW.

We see that regardless of $R o$, large-scale effects tend to stabilise states, especially strongly cyclonic ones. In addition, we see that for large- $\gamma$ states, the location of $\lambda_{s}$ becomes $\gamma$-independent.

The determination of $\lambda_{s}$ for anticyclonic states at $\gamma=0.25$ has proved difficult, as in addition to being affected by an elliptical wavenumber-3 instability (hereafter the mode-3 instability, see Dritschel 1986) which leads to the state shedding a filament from one of its tips, small-scale disturbances may develop on the vortex boundary, which grow in time and eventually lead to the state becoming unstable. The form of $\epsilon_{b}(t)$, shown in figure 7 , is very different for such states. As seen from the thin line in the figure, unstable states have a sharp, clearly defined jump in $\epsilon_{b}$, while weaklyunstable states exhibit a slow growth of $\epsilon_{b}$ up to and after the time the first filament is shed, which is marked by the thin and bold dashed vertical lines. A jump in $\epsilon_{b}$ may occur in the weakly-unstable state once the small-scale filaments shed by the vortex have interacted with its boundary to such an extent that a larger filament is shed. For comparison, in the figure we also show $\epsilon_{b}$ for a state which is stable for all time (grey line), and we see that the weakly-unstable state very gradually begins to depart from these values before a filament is shed. We find this type of 'weak' instability occurring even at large aspect ratios, and it may be surrounded by extensive regions of stable aspect ratios. We therefore classify these weakly-unstable states as stable.

A clear difference in the stability of cyclones and anticyclones is visible in figure 6 . Many previous studies have examined the cyclone-anticyclone asymmetry, and it has been reported that in decaying SW turbulence and in more realistic oceanic flows, large anticyclonic structures are more prevalent than their cyclonic counterparts (Olson 1991; Arai \& Yamagata 1994; Polvani et al. 1994). Furthermore, cyclones are generally found to be more deformed than anticyclones (Aristegui et al. 1994). Although anticyclones appear to be more long-lived, there is no evident asymmetry between the distribution of cyclones and anticyclones (Olson 1991). The results of 


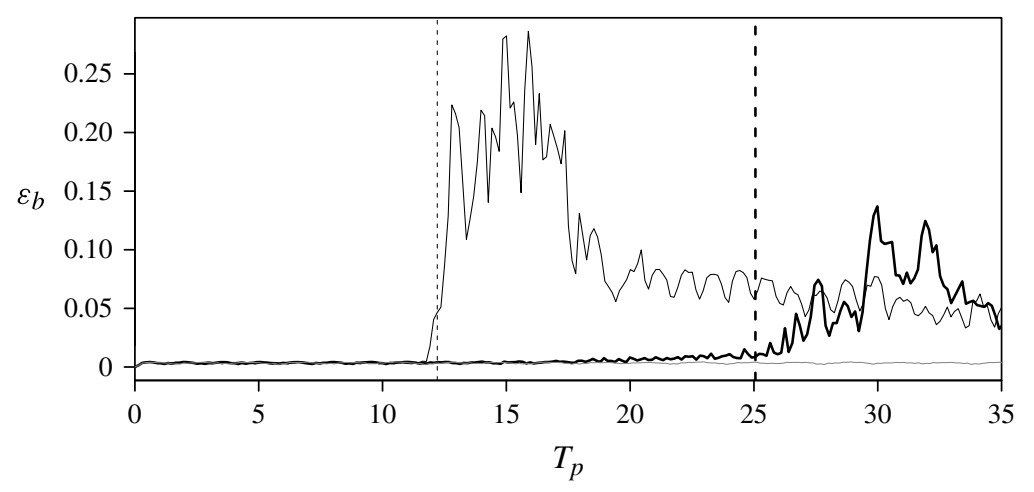

FIGURE 7. The steadiness parameter $\epsilon_{b}$ as a function of time for the case $(\gamma, R o)=$ $(0.25,-0.1)$ near $\lambda=\lambda_{c}$. We see an unstable state at $\lambda=0.315$ (thin line), a stable state with a weak instability at $\lambda=0.325$ (bold line) and a state stable for all time at $\lambda=0.340$ (grey line). The times at which the first filament is shed are indicated with the two dashed lines.

our study confirm these observations. Across all $\gamma$, we find that cyclones are able to sustain greater deformations than anticyclones while remaining stable.

Anticyclones are in general believed to be more 'stable' than cyclones (see e.g. Stegner \& Dritschel 2000; Baey \& Carton 2002). We find the opposite: ageostrophic effects tend to stabilise cyclones and destabilise anticyclones. However, it is important to keep in mind that when comparing cyclonic and anticyclonic motions, it is necessary to define what is meant by two states which are 'equivalent', as cyclones and anticyclones cannot simultaneously have identical (but oppositely-signed) velocities, PV and height anomalies.

Here, we say two states are 'equivalent' when they have the same magnitude of Rossby number $|R o|$ (which defines the PV anomaly), size relative to the Rossby deformation length and aspect ratio $\lambda$. Note that the size of the vortex $L$ is calculated from the size it has in QGSW, where it has a prescribed area of $\pi$, giving $L=1$. After the initial ramping of the PV anomaly, the vortex area generally differs from $\pi$, though never by more than a few per cent across the entire parameter space investigated. The important point is that the vortex shape, circulation and mass distribution is uniquely determined from the QGSW state and the PV ramping, and hence these vortex properties are reproducible. Stegner \& Dritschel (2000) studied circular, isolated, distributed SW vortices and chose to examine 'equivalent' vortices which shared the Burger number (recall that this is our $\gamma^{-2}$ ) and (oppositely-signed) relative vorticity profiles, hence the differences in their conclusions.

To the best of the authors' knowledge, a careful study of the cyclone-anticyclone asymmetry, which spans a wide parameter space and where the 'equivalence' between two states is clearly defined has not been previously performed. We find that cyclones are stable for a wider range of the parameter regime than anticyclones, and that largescale effects tend to stabilise them more easily than anticyclones. We also find that for cyclones, a strong instability which results in the break-up of the vortex may not exist at all, and instead only a weak instability in the form of vacillations occurs. Anticyclones on the other hand are stable for a smaller portion of the parameter space, and, especially at large $|R o|$, are less likely to experience vacillations.

For completeness, in figure 8 we show the asymmetries which arise in the height field for vortices which we call 'equivalent'. We see that at small $\gamma=0.25(a)$, 
(a)

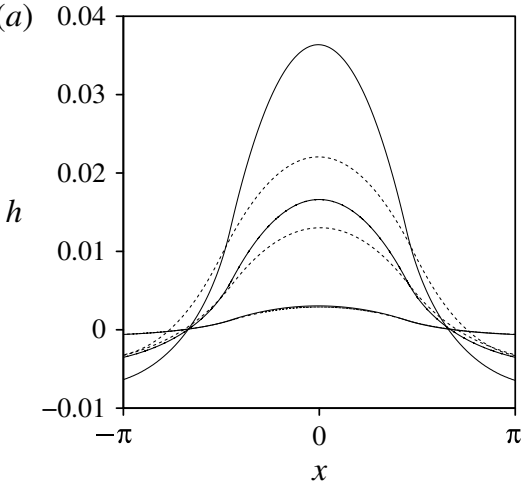

(b)

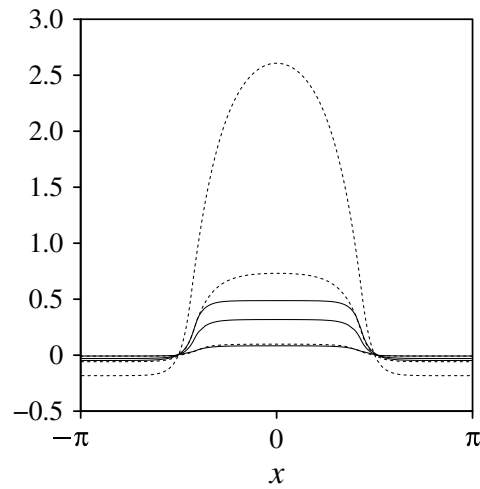

FIGURE 8 . The cross-section of the height anomaly $-h$ for cyclonic (thin lines) and $h$ for anticyclonic (dashed lines) states at $\lambda=0.5$. Here, we show the value at $y=0$ between $-\pi \leqslant x \leqslant \pi$ of states which have been rotated to be aligned with the $x$-axis at the end of the PV ramp period. We see $\gamma=0.25(a)$ and $\gamma=6(b)$, each at $|R o|=0.1,0.5$ and 1 , with lower $|R o|$ states having smaller $h$ (states at $|R o|=0.1$ are nearly indistinguishable). Note the different scales of the vertical axis in each of the panels.

cyclones (thin lines) have larger height anomalies than anticyclones (dashed lines), and although the shapes of the height cross-sections are similar between the two types of motion, anticyclones are more 'sprawled' than cyclones. (Note that in the figure we plot $-h$ for anticyclones.) On the other hand, for large $\gamma=6(b)$ anticyclones have larger height anomalies, and there is a difference in shape between the two types of motions. Cyclones have a flat height profile in the middle with a steep rise near their edge, whereas anticyclones have a parabolic cross-section. These difference are most pronounced at large $|R o|$, at which also the asymmetries in the stability of the two types of motion are most apparent. See also figure 5 for an illustration of the asymmetries in the masses of cyclones and anticyclones at the boundary of stability.

\section{Types of evolution}

Below, we describe the types of unstable evolution that vortices exhibit near $\lambda_{c}$, and where they occur in the $\gamma-R o$ parameter space. We find four distinct types of instability regimes, which, following the classification made by Płotka \& Dritschel (2012) in QGSW, we call type 1 instability for vacillating states, type 2 instability for states which shed a filament and type $3 \mathrm{i}$ and $3 \mathrm{ii}$ instabilities for states which split into two vortices of unequal or equal size, respectively.

We first show a few examples of the evolution of each of the instability types. In figure 9 an example of the type 1 instability is shown: a weakly unstable, vacillating state having $(\gamma, R o)=(6,0.8)$ at $\lambda=0.005$, the smallest aspect ratio examined. As time progresses, we see that the state increases its initial aspect ratio $\lambda_{i}$ (figure $9 a$ ) until some maximum value of a vacillation cycle $\lambda_{\max }^{c y c}$ (figure $9 b$ ), before decreasing it again to $\lambda_{\text {min }}^{c y c}$ (figure 9c). After the first few vacillation cycles where it is comparable to $\lambda_{i}, \lambda_{\min }^{c y c}$ settles down to a constant value which is larger than $\lambda_{i}$, while the value of $\lambda_{\max }^{c y c}$ has similar values for the entire duration of the simulation. As visible in figure $9(b)$ there are large-amplitude waves travelling on the boundary of the equilibrium, which obey a modified Korteweg-de Vries equation to leading order in $L_{D}$ (Nycander et al. 1993). The behaviour described above is typical of all vacillating states, although as 
(a)

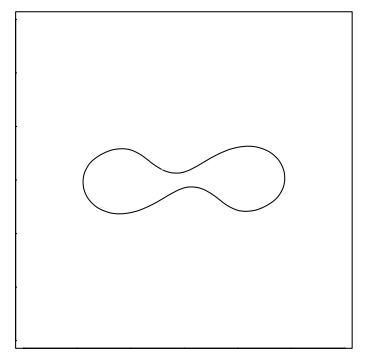

(b)

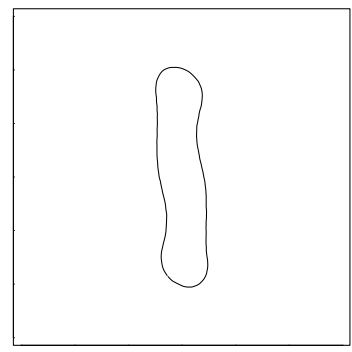

(c)

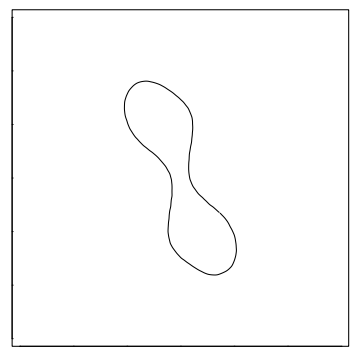

FIGURE 9. An example of a vacillating state (type 1 instability) having $(\gamma, R o, \lambda)=$ $(6,0.8,0.005)$. We see time $t=0.84 T_{p}$ after the PV ramping period has finished $(a)$, and times $t=19.49 T_{p}$ and $t=22.85 T_{p}$ when the state reaches a maximum $(b)$ and minimum (c) aspect ratio during a vacillation cycle. Here and in subsequent figures we see contours of the PV anomaly $q^{\prime}$, with $|x|,|y| \leqslant \pi$.

(a)

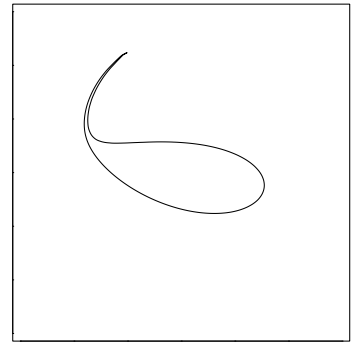

(d)

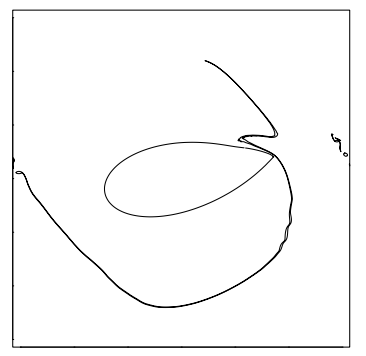

(b)

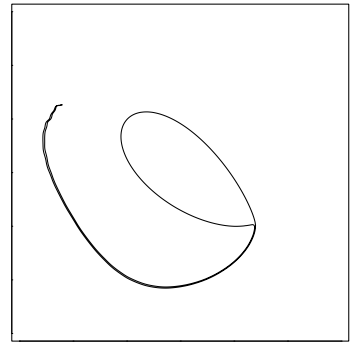

(e)

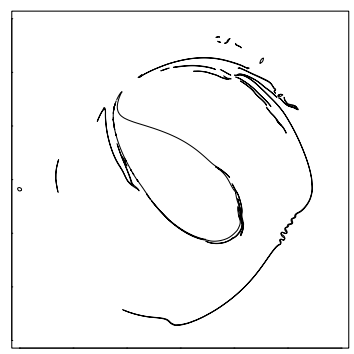

(c)

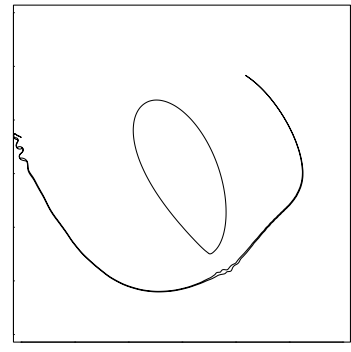

(f)

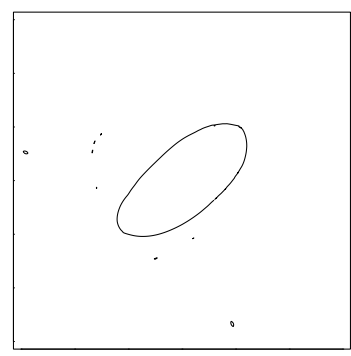

FIGURE 10. An example of a state having $(\gamma, R o, \lambda)=(0.25,0.7,0.310)$, which sheds a filament (type 2 instability). We see the state at times $(a) t=21.22 T_{p}$, (b) $t=21.73 T_{p}$, (c) $t=22.24 T_{p},(d) t=22.50 T_{p}$, (e) $t=23.52 T_{p}$ and $(f) t=30.68 T_{p}$.

$\lambda_{i}$ increases towards $\lambda_{s}$, both the amplitude of the vacillation and that of the waves travelling on the equilibrium boundary decreases. As seen in figure 6 in the previous section, this sort of instability occurs only in states having $\gamma \geqslant 1$.

Figure 10 shows an example of the type 2 instability: for a state having $(\gamma, R o)=$ $(0.25,0.7)$ at $\lambda=\lambda_{c}=0.310$, which sheds a filament in a mode- 3 instability. As seen from figure $10(d, e)$, further interactions between the original state and the filamentary debris can occur after the filament has been shed. The state eventually evolves towards a quasi-steady state, which has small values of $\epsilon_{b}$, albeit larger ones than initially, as discussed in $\S 3$.

Parts of the filament may roll up to form small satellite vortices (figure 10f). For states with $\gamma=0.25$, these satellite vortices are negligible in size and make up less than $0.3 \%$ of the total mass held by vortices in the domain. At higher $\gamma$ where this 
(a)

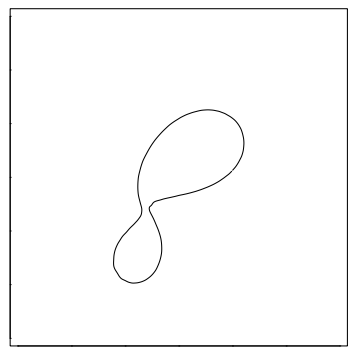

(b)

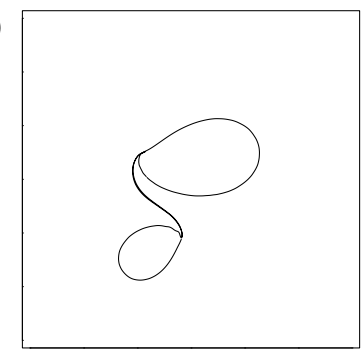

(c)

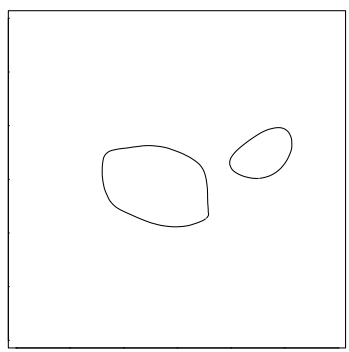

FIGURE 11. An example of a state having $(\gamma, \operatorname{Ro}, \lambda)=(4,-0.8,0.150)$, which undergoes a split into two asymmetric vortices (type $3 \mathrm{i}$ instability). We see the state right before the split at $t=22.93 T_{p}(a)$, during the split at $t=23.07 T_{p}(b)$ and what the state looks like at a late time $t=54.93 T_{p}(c)$.

instability occurs, the largest of the small vortices can hold up to $2.8 \%$ of the mass, and the state resembles one having undergone the type $3 \mathrm{i}$ instability.

There is a smooth transition between the different types of instabilities occurring, as seen in figure 13 showing that more than one type of instability may occur at a fixed $(\gamma, R o)$ at different nearby $\lambda$ (this is discussed further below). In addition, our results are consistent with those of Waugh \& Dritschel (1991), who found that strips of PV are more likely to roll up to form vortices as the interaction range decreases, i.e. for increasing $\gamma$.

In figure 11 we show an example of the type $3 \mathrm{i}$ instability for an anticyclonic state having $(\gamma, R o)=(4,-0.8)$ at $\lambda=\lambda_{c}=0.150$, which splits into two vortices of unequal size. During the onset of instability, the state exhibits behaviour similar to that of the type 2 instability, where it looks like it is about to shed a filament. There is a competition between the mode- 3 and mode- 4 instabilities, and the state pinches off a small vortex, which, especially for $1 \leqslant \gamma<3$, may further interact with the larger vortex before reaching a final quasi-steady state in which the two vortices corotate. Although not occurring in the case shown in the figure, as in the type 2 instability regime, small satellite vortices may roll up from the filaments shed during the split. Apart from the case $(\gamma, R o, \lambda)=(1,-0.1,0.230)$ where the largest satellite vortex contains $3.5 \%$ of the total mass held by the vortices in the domain (this is compared with $5 \%$ of the mass held by the second-largest vortex in the domain), satellite vortices normally contain less than $0.6 \%$ of the mass.

Finally, in figure 12 we show an example of a state having $(\gamma, R o)=(4,-0.2)$ at $\lambda=\lambda_{c}=0.065$, which splits into two vortices of equal size. The state rapidly decreases its aspect ratio and splits, shedding tiny filaments which, as seen in figure $12(c)$, roll up into very small vortices containing $\ll 1 \%$ of the total mass held by vortices. Because of its resemblance to type $3 \mathrm{i}$ instability, we call this type 3 ii instability.

We next present a quantitative measure for dividing unstable vortex behaviour into the four instability regimes described above. In $\S 3$ we have already discussed how we determine whether a state is vacillating, and here we instead focus on the evolution types of states which undergo break-up. As the end state is generally composed of two larger vortices in a sea of filamentary debris, we perform the classification based on the ratio of the masses of the two largest patches of PV in the domain at the end of the simulation, $M_{r}=M_{2} / M_{1}$, where the subscripts 1 and 2 denote the largest and second-largest vortices in the domain, respectively. We say that a state undergoes type 2 instability, filamentation, when $M_{r}<0.03$. The type $3 \mathrm{i}$ and $3 \mathrm{ii}$ 
(a)

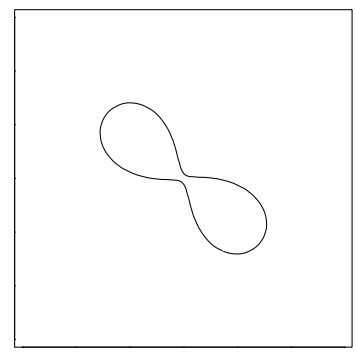

(b)

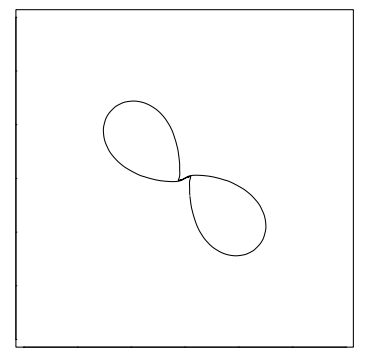

(c)

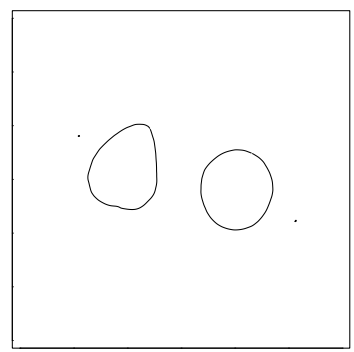

FIGURE 12. An example of a state having $(\gamma, \operatorname{Ro}, \lambda)=(4,-0.2,0.065)$, which undergoes a split into two symmetric vortices (type 3ii instability). We see two times right before the split, $t=2.15 T_{p}(a)$ and $t=2.28 T_{p}(b)$, and what the state looks like at a late time $t=38.50 T_{p}(c)$.

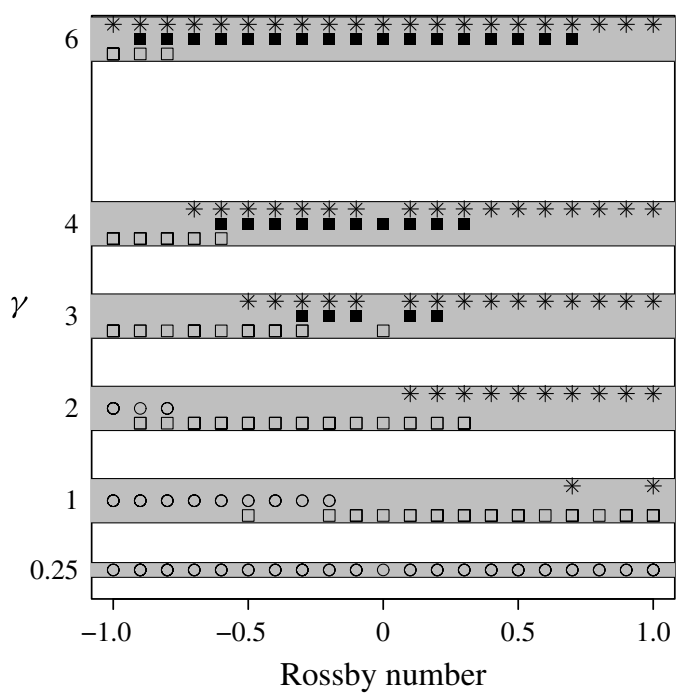

FIGURE 13. A summary of the instability types at each $\gamma$ classified by their end mass shown at each $R o$ for aspect ratios near $\lambda_{c}$. The instability types shown are: type 1, vacillations (asterisks); type 2, filamentation with $M_{r}<0.03$ (circles); type 3i, asymmetric split with $0.03 \leqslant M_{r}<0.99$ (empty squares); and type 3ii, symmetric split with $M_{r} \geqslant 0.99$ (filled-in squares). Note that at each $\gamma$ and $R o$ multiple instability mechanisms may be possible, which are shown on separate lines at each $\gamma$. We also show the $R o=0$ results, as found by Płotka \& Dritschel (2012).

instability regimes, asymmetric and symmetric splitting, occur if $0.03 \leqslant M_{r}<0.99$ and $M_{r} \geqslant 0.99$, respectively.

Figure 13 shows a summary of where the different instabilities occur in the $\gamma-R o$ parameter plane, with type 1 instability marked by asterisks, type 2 instability by circles, and types $3 \mathrm{i}$ and $3 \mathrm{ii}$ by empty squares and filled-in squares, respectively. Note, several types of instability can occur at the same $(\gamma, R o)$ value; these instabilities occur on vortices with different $\lambda$ near $\lambda_{c}$. We see that for states with $\gamma<1$, the type 2 instability is the only one occurring, while at large $\gamma>2$ it is completely absent. In the intermediate vortex-size range $1 \leqslant \gamma \leqslant 2$, this type of instability only 
occurs in anticyclones, and only for increasingly large $|R o|$ as $\gamma$ increases. The type 1 and 3 instabilities are typical of the large- $\gamma$ states. Type $3 \mathrm{i}$ instability occurs at smaller $\gamma$, where it is more common in cyclones, but as $\gamma$ increases, it starts affecting a narrower range of increasingly small $R o$ : at $\gamma=1$ it affects states with $-0.5 \leqslant R o \leqslant 1$, whereas at $\gamma=6$ it only affects states with $-1 \leqslant R o \leqslant-0.8$. Type 3ii instability occurs only for large $\gamma \geqslant 3$, and similarly to type $3 \mathrm{i}$ instability, at smaller $\gamma$ it occurs mainly in cyclones, and in anticyclones with small $|R o|$. As $\gamma$ increases it begins to affect states with larger $|R o|$. This is also true of the type 1 instability, which, as $\gamma$ increases, begins to affect a wider range of Ro. As mentioned above, as $\gamma$ increases, there is a smooth transition from the type 2 to type $3 \mathrm{i}$ instabilities, with the filaments shed rolling up to form increasingly large vortices, and from the type $3 \mathrm{i}$ to 3ii instabilities, with the smaller vortex gradually becoming larger until the mode-4 instability, resulting in a symmetric split of the state, dominates over the mode-3 one which causes a filament to be shed from one of the tips of the vortex.

\section{Conclusions}

We have examined the form and stability of vortex-patch relative quasi-equilibria in the single-layer $f$-plane $\mathrm{SW}$ model of geophysical fluid dynamics. These states depend on three parameters: the ratio $\gamma$ between their mean radius $L$ and the Rossby deformation length $L_{D}$, their aspect ratio $\lambda$ and their strength relative to the background rotation, measured by the PV-based Rossby number $R o=q^{\prime} / f$.

The states we generate in this model problem are not strictly in equilibrium, since in SW, even in initially well-balanced flows, there is always some degree of IGW generation. By defining a 'steadiness' parameter, which measures how closely aligned the contours of PV (defining the boundary of the vortex) and those of the Bernoulli pressure function are, we show that both the stable and unstable states generated are steady, which is further confirmed by the long times for which the stable states remain unchanged in shape; indeed, they have less than a $5 \%$ average variation in $\lambda$ over the duration of the simulation. For simplicity, we refer to the quasi-equilibria as just equilibria.

We find the stable aspect ratio $\lambda_{s}$, depending on both $\gamma$ and $R o$, at which equilibria cease to be steady. The instability can either be weak, occurring at $\lambda_{s}=\lambda_{v}$, where the state vacillates around the steady state by going through cycles of increasing and decreasing aspect ratio, or strong, at $\lambda_{s}=\lambda_{c}$, where the vortex undergoes a drastic change in shape, often involving a break-up into two or more parts. For both the weakly-unstable vacillating states, and for the strongly unstable ones after they have settled down following instability, the flow is often close to equilibrium. States for which $\lambda_{s}=\lambda_{v}$ may still exist nearby states having $\lambda=\lambda_{c}$ such that for any $\lambda<\lambda_{c}$ the vortex experiences a strong instability. Multiple forms of instability may occur for nearby values of $\lambda$.

Large-scale effects (large $\gamma$ values) stabilise vortices, regardless of their strengths $(R o)$. These large-scale stabilising effects are most pronounced for large-Ro states, where the location of $\lambda_{s}$ for states with $\gamma \gtrsim 1$ becomes $\gamma$ - and $R o$-independent. We find that for states with $\gamma \gtrsim 3$ and $R o \gtrsim-0.6$, the location of $\lambda_{s}$ becomes $\gamma$-independent, and for $R o \gtrsim 0.8$ also $R o$-independent. This difference in behaviour between small- $\gamma$ and large- $\gamma$ states is also visible in the types of unstable evolution they undergo near $\lambda_{c}$ : small- $\gamma$ states are affected by the mode- 3 instability, which causes them to shed a filament from one of the tips of the vortex, whereas for large- $\gamma$ states the most unstable mode is mode- 4 , which causes a nearly-symmetric split of 
the vortex. In the intermediate- $\gamma$ range there is a competition between the two modes, resulting in a split of the vortex into two parts of unequal size.

By calling two states which share $\gamma,|R o|$ and $\lambda$ 'equivalent', we find a clear asymmetry in the stability of cyclonic $(R o>0)$ and anticyclonic $(R o<0)$ equilibria. Cyclones can sustain greater deformations than anticyclones before experiencing an instability. In fact, for large enough $\gamma$ and $R o$ (i.e. $\gamma \gtrsim 2, R o \gtrsim 0.5$ ) they may not experience a 'strong' instability at all, even for $\lambda$ close to 0 . They rather undergo a weak instability, vacillating around an underlying equilibrium state. In general, cyclones are more prone to experiencing such a weak instability, with it being completely absent for anticyclones with large enough $|R o|$ at $\gamma$ between 2 and 4 . In short, ageostrophic motions stabilise cyclones and destabilise anticyclones. Both types of vortices undergo the same main types of unstable evolution, although the mode-4 instability dominates over the mode-3 one in cyclones at smaller $\gamma$ than in anticyclones. This cyclone-anticyclone asymmetry is the opposite of what is generally reported in the literature. This, we believe, is due to the difficulty in defining 'equivalent' cyclones and anticyclones.

We have found a rapid return of the flow to a quasi-steady state after an instability has taken place. This quasi-steady state often has the form of two corotating vortices of equal or unequal size. Płotka \& Dritschel (2013) studied equilibrium forms of such structures in the fully-balanced QGSW context, and it would be of interest to extend their work to SW to see what role ageostrophic effects play in the (quasi-)equilibrium forms of such states. This would also allow us to gain insight into the nature of the quasi-steady states arising in this study, and to check whether transitions between equilibrium solutions take place.

Although the single-layer SW approximation provides insight into realistic geophysical motions, the assumption of a lower layer of infinite depth may not always be physical. Polvani et al. (1989) has shown that a finite-depth lower layer greatly influences the form of QGSW equilibria, with large-scale effects not playing such an important role in this set-up. It would be of interest to see how this is affected in a model which allows ageostrophic motions.

\section{Acknowledgements}

Hanna Płotka would like to acknowledge the support of a UK Natural Environment Research Council studentship.

\section{Appendix}

The 'optimal PV balance' procedure Viúdez \& Dritschel (2004) starts with a flow at rest, and artificially ramps up the PV anomaly of each fluid particle by multiplying it by a ramp function $T(\tau)=(1 / 2)\left[1-\cos \left(\pi \tau / \Delta_{\tau}\right)\right]$, which varies smoothly between 0 and 1 from the beginning $(\tau=0)$ to the end $\left(\tau=\Delta_{\tau}\right)$ of the ramping period. Apart from the ramping of $\mathrm{PV}$, the full $\mathrm{SW}$ equations are solved. The 'target configuration', having only minimal amounts of imbalance if a sufficiently long $\Delta_{\tau}$ is considered (in practice, exceeding 3 inertial time periods $T_{i p}=2 \pi / f$ ), is determined through a series of backward and forward iterations, where $\tau$ plays the role of the iteration parameter. The procedure does not enforce any specific balance conditions (such as e.g. geostrophic balance), but does depend on the choice of $\Delta_{\tau}$. Because of the sometimes very large $q^{\prime}$ considered in this study, convergence to the target configuration for long enough $\Delta_{\tau}$ to truly minimise the presence of IGWs is not possible. Therefore, instead of performing a series of iterations until the 


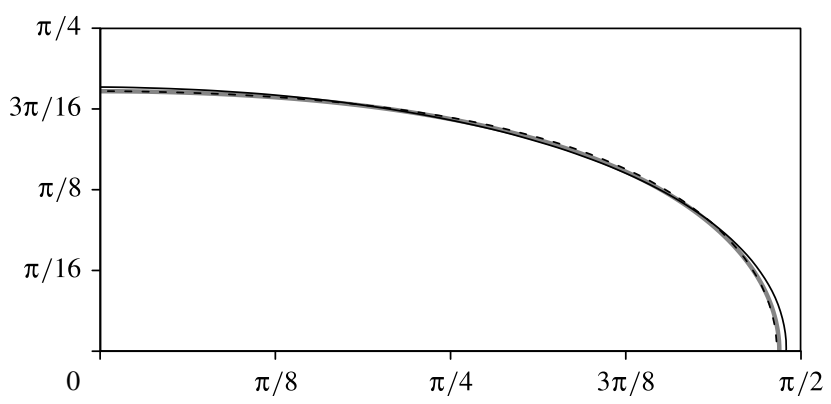

FIGURE 14. PV contours of states with $\gamma=1$ and $\lambda=0.400$ at time $t=\Delta_{\tau}=10 T_{i p}$ at the end of the ramping period. We show equilibria with three different $R o$ : the initial QGSW state with $R o=0$ (grey line), a cyclonic state with $R o=0.5$ (dashed black line) and an anticyclonic state with $R o=-0.5$ (solid black line). Note that the cyclonic and anticyclonic states have been rotated to be aligned with the $x$-axis.

target configuration in which the "balanced' PV contours coincide exactly with those specified initially, we perform only one forward iteration, allowing the initial contours to adjust to the flow. This allows us to consider $\Delta_{\tau}=10 T_{i p}$, which is sufficiently long for the generation of IGWs to be kept to a minimum while the flow adjusts itself around the PV anomaly. Once the prescribed PV anomaly is reached, the PV is thereafter conserved and allowed to evolve freely. The minimally-unbalanced flows initialised in this way allow for the study of equilibria, and any destructive effects of artificially generated IGWs are attenuated.

Figure 14 shows the original contours of PV for a QGSW state (the grey line in the figure) and those of a cyclonic (dashed black line) and anticyclonic one (solid black line) with $(\gamma,|R o|)=(1,0.5)$ and $\lambda=0.400$ at $t=\Delta_{\tau}$ at the end of the ramping period. During the ramp, the SW states undergo rotation and are here shown rotated back to be aligned with the $x$-axis. For clarity, in the figure we only show the form of the contours in the first quadrant, but qualitatively they look similar in the remaining three quadrants. From figure 14 we see that at the end of the ramping period, despite being allowed to evolve freely, the SW contours still closely resemble the QGSW one. This is especially so for the cyclonic state. Despite over-estimating the locations of the extrema of the contour, the anticyclonic state is still remarkably close to the QGSW one. This is especially astonishing since we are considering $R o=-0.5$, where a considerable free-surface deformation (of over $15 \%$ of the fluid depth) has taken place. Therefore, we henceforth make the approximation that the QGSW value of $\lambda$ is the same as the SW one at the end of the ramping period.

\section{REFERENCES}

Arai, M. \& Yamagata, T. 1994 Asymmetric evolution of eddies in rotating shallow water. Chaos 4 (1), 163-175.

Aristegui, J., Sangrá, P., Hernandez-león, S., Canton, M., Hernández-Guerra, A. \& Kerling, J. L. 1994 Island-induced eddies in the Canary islands. Deep-Sea Res. 41, 1509-1525.

BAEY, J.-M. \& CARTON, X. 2002 Vortex multipoles in two-layer rotating shallow-water flows. J. Fluid Mech. 460, 151-175.

CARton, X. 2001 Hydrodynamical modelling of oceanic vortices. Surv. Geophys. 22, 179-263. 
Cushman-Roisin, B. 1987 Exact analytical solutions for the elliptical vortices of the shallow-water equations. Tellus A 39, 235-244.

Cushman-Roisin, B., Heil, W. H. \& Nof, D. 1985 Oscillations and rotations of elliptical warmcore rings. J. Geophys. Res. 90 (C6), 11756-11764.

Cushman-Roisin, B. \& TANG, B. 1990 Geostrophic turbulence and emergence of eddies beyond the radius of deformation. J. Phys. Oceanogr. 20, 97-113.

Deem, G. S. \& Zabusky, N. J. $1978 a$ Stationary 'V-states', interactions, recurrence, and breaking. In Solitons in Action (ed. K. Lonngren \& A. Scott), pp. 277-293. Academic Press.

Deem, G. S. \& ZABusky, N. J. $1978 b$ Vortex waves: stationary 'V-states', interactions, recurrence, and breaking. Phys. Rev. Lett. 40, 859-862.

Dritschel, D. G. 1985 The stability and energetics of corotating uniform vortices. J. Fluid Mech. 157, 95-134.

Dritschel, D. G. 1986 The nonlinear evolution of rotating configurations of uniform vorticity. J. Fluid Mech. 172, 157-182.

DritsChel, D. G. 1995 A general theory for two-dimensional vortex interactions. J. Fluid Mech. 293, 269-303.

Dritschel, D. G. \& Ambaum, M. H. P. 1997 A contour-advective semi-Lagrangian algorithm for the simulation of fine-scale conservative dynamical fields. Q. J. R. Meteorol. Soc. 123, 1097-1130.

Dritschel, D. G., Polvani, L. M. \& Mohebalhojeh, A. R. 1999 The contour-advective semiLagrangian algorithm for the shallow water equations. Mon. Weath. Rev. 127, 1551-1565.

Dritschel, D. G. \& ViúdeZ, Á 2003 A balanced approach to modelling rotating stably stratified geophysical flows. J. Fluid Mech. 488, 123-150.

FORD, R. 1994 The response of a rotating ellipse of uniform potential vorticity to gravity wave radiation. Phys. Fluids 6 (11), 3694-3704.

Ford, R., McIntyre, M. E. \& Norton, W. A. 2000 Balance and the slow quasimanifold: some explicit results. J. Atmos. Sci. 57, 1236-1254.

Garate-Lopez, I., Hueso, R., Sánchez-Lavega, A., Peralta, J., Piccioni, G. \& Drossart, P. 2013 A chaotic long-lived vortex at the southern pole of Venus. Nature Geosci. 6, 254-257.

GRIFFITHS, R. W. \& Hopfinger, E. J. 1986 Experiments with baroclinic vortex pairs in a rotating fluid. J. Fluid Mech. 173, 501-518.

Griffiths, R. W. \& Hopfinger, E. J. 1987 Coalescing of geostrophic vortices. J. Fluid Mech. 178, 73-97.

Hoskins, B. J., McIntyre, M. E. \& Robertson, A. W. 1985 On the use and significance of isentropic potential vorticity maps. Q. J. R. Meteorol. Soc. 111, 877-946.

KIRChHOfF, G. R. 1876 Vorlesungen über mathematische Physik. Mechanik. B. G. Teubner.

Kizner, Z., Berson, D. \& Khvoles, R. 2002 Baroclinic modon equilibria on the beta-plane: stability and transitions. J. Fluid Mech. 468, 239-270.

Kizner, Z., Reznik, G., Fridman, B., Khvoles, R. \& MCWilliams, J. C. 2008 Shallow-water modons on the $f$-plane. J. Fluid Mech. 603, 305-329.

Luzzatto-Fegiz, P. \& Williamson, C. H. K. 2010 Stability of elliptical vortices from 'imperfectvelocity-impulse' diagrams. Theor. Comput. Fluid Dyn. 24, 181-188.

Luzzatto-Fegiz, P. \& Williamson, C. H. K. 2011 An efficient and general numerical method to compute steady uniform vortices. J. Comput. Phys. 230, 6495-6511.

Makarov, V. G. \& Kizner, Z. 2011 Stability and evolution of uniform-vorticity dipoles. J. Fluid Mech. 672, 307-325.

Makarov, V. G., Sokolovskiy, M. A. \& Kizner, Z. 2012 Doubly symmetric finite-core heton equilibria. J. Fluid Mech. 708, 397-417.

Malanotte-Rizzoli, P. 1982 Planetary solitary waves in geophysical flows. Adv. Geophys. 24, $147-224$.

Mcintyre, M. E. \& Norton, W. A. 2000 Potential vorticity inversion on a hemisphere. J. Atmos. Sci. 57, 1214-1235.

MCKiver, W. J. \& Dritschel, D. G. 2008 Balance in non-hydrostatic rotating stratified turbulence. J. Fluid Mech. 596, 201-219. 
McWilliams, J. C. 1984 The emergence of isolated coherent vortices in turbulent flow. J. Fluid Mech. 146, 21-43.

McWilliams, J. C. 1985 Submesoscale, coherent vortices in the ocean. Rev. Geophys. 23, 165-182.

Mohebalhojeh, A. R. 2002 On shallow water potential vorticity inversion by Rossby-number expansions. Q. J. R. Meteorol. Soc. 128, 679-694.

Mohebalhojeh, A. R. \& Dritschel, D. G. 2000 On the representation of gravity waves in numerical models of the shallow-water equations. Q. J. R. Meteorol. Soc. 126, 669-688.

Nycander, J., Dritschel, D. G. \& Sutyrin, G. G. 1993 The dynamics of long frontal waves in the shallow water equations. Phys. Fluids A 5, 1089-1091.

Olson, D. B. 1991 Rings in the ocean. Annu. Rev. Earth Planet. Sci. 19, 283-311.

Pedlosky, J. 1979 Geophysical Fluid Dynamics. Springer-Verlag.

PIERREHUMBert, R. T. 1980 A family of steady, translating vortex pairs with distributed vorticity. J. Fluid Mech. 99, 129-144.

PŁotKA, H. \& Dritschel, D. G. 2012 Quasi-geostrophic shallow-water vortex-patch equilibria and their stability. Geophys. Astrophys. Fluid Dyn. 106 (6), 574-595.

PŁotKA, H. \& DRITsChel, D. G. 2013 Quasi-geostrophic shallow-water doubly-connected vortex equilibria and their stability. J. Fluid Mech. 723, 40-68.

Polvani, L. M. 1988 Geostrophic vortex dynamics. PhD thesis, MIT/WHOI WHOI-88-48.

Polvani, L. M., MCWilliams, J. C., Spall, M. A. \& Ford, R. 1994 The coherent structures of shallow-water turbulence: deformation-radius effects, cyclone/anticyclone asymmetry and gravity-wave generation. Chaos 4 (2), 177-186.

Polvani, L. M., Zabusky, N. J. \& Flierl, G. R. 1989 Two-layer geostrophic vortex dynamics. Part 1. Upper-layer V-states and merger. J. Fluid Mech. 205, 215-242.

RIPA, P. 1987 On the stability of elliptical vortex solutions of the shallow-water equations. J. Fluid Mech. 183, 343-363.

SAfFMAN, P. G. \& SzETo, R. 1980 Equilibrium shapes of a pair of equal uniform vortices. Phys. Fluids 23, 2339-2342.

SMith, R. K. \& DRITSChel, D. G. 2006 Revisiting the Rossby-Haurwitz wave test case with contour advection. J. Comput. Phys. 217, 473-484.

Stegner, A. \& Dritschel, D. G. 2000 A numerical investigation of the stability of isolated shallow water vortices. J. Phys. Oceanogr. 30, 2562-2573.

Thomson, W. 1875 Vortex statics. Math. Phys. Pap. IV, 115-128.

Vallis, G. K. 2008 Atmospheric and Oceanic Fluid Dynamics. Cambridge University Press.

VIÚdEZ, Á \& Dritschel, D. G. 2004 Optimal potential vorticity balance of geophysical flows. J. Fluid Mech. 521, 343-352.

Waugh, D. W. 1992 The efficiency of symmetric vortex merger. Phys. Fluids A 4, 1745-1758.

WAugh, D. W. \& Dritschel, D. G. 1991 The stability of filamentary vorticity in two-dimensional geophysical vortex-dynamics models. J. Fluid Mech. 231, 575-598.

Waugh, D. W. \& Polvani, L. M. 2010 Stratospheric polar vortices. In The Stratosphere: Dynamics, Chemistry, and Transport (ed. L. M. Polvani, A. H. Sobel \& D. W. Waugh), Geophys. Monogr. Ser., vol. 190, pp. 43-57. AGU.

YASUDA, I. 1995 Geostrophic vortex merger and streamer development in the ocean with special reference to the merger of Kuroshio warm core rings. J. Phys. Oceanogr. 25, 979-996. 